\title{
$\begin{array}{lllllllllllllllll}\text { S } & \mathbf{A} & \mathbf{M} & \mathbf{M} & \mathbf{L} & \mathbf{U} & \mathbf{N} & \mathbf{G} & & \mathbf{T} & \mathbf{U} & \mathbf{S} & \mathbf{C} & \mathbf{U} & \mathbf{L} & \mathbf{U} & \mathbf{M}\end{array}$
}

In Tusculum, vor den Toren Roms,

hatte Cicero sein Landhaus.

In Zeiten der Muße, aber auch

der politischen Isolation, zog er sich

dorthin zurück.

Tusculum wurde zum Inbegriff für

Refugium, für Muße, für wertvolle

Fluchten aus einem fordernden Alltag.

In der ersten Phase des

Rückzugs aus der Politik schrieb

Cicero in Tusculum die sogenannten

Tuskulanen, eine lateinische

Einführung in die Welt

der (griechischen) Philosophie.

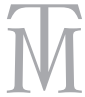

Herausgeber:

Niklas Holzberg

Rainer Nickel

Karl-Wilhelm Weeber

Bernhard Zimmermann 



\section{PETRONIUS ARBITER}

\section{SATYRISCHE GESCHICHTEN}

\section{SATYRICA}

Lateinisch - deutsch

Herausgegeben und übersetzt von Niklas Holzberg 
Bibliografische Information der Deutschen Nationalbibliothek Die Deutsche Nationalbibliothek verzeichnet diese Publikation in der Deutschen Nationalbibliografie; detaillierte bibliografische Daten sind im Internet über http://dnb.d-nb.de abrufbar.

(C) Akademie Verlag GmbH, Berlin 2013

Ein Wissenschaftsverlag der Oldenbourg Gruppe

www.akademie-verlag.de

Das Werk einschließlich aller Abbildungen ist urheberrechtlich geschützt. Jede Verwertung außerhalb der Grenzen des Urheberrechtsgesetzes ist ohne Zustimmung des Verlages unzulässig und strafbar. Das gilt insbesondere für Vervielfältigungen, Übersetzungen, Mikroverfilmungen und die Einspeicherung und Bearbeitung in elektronischen Systemen.

Einbandgestaltung: Gabriele Burde, Berlin

Satz: Dörlemann Satz, Lemförde

Druck und Bindung: Pustet, Regensburg

Dieses Papier ist alterungsbeständig nach DIN/ISO 9706.

ISBN 978-3-05-006016-3 